\title{
Effects of Waveform and Cycle Period on Corrosion-Fatigue Crack Growth in Cathodically Protected High-Strength Steels
}

\author{
Mark Knop ${ }^{1,2, a^{*}}$, Nick Birbilis ${ }^{2, b}$ and Stan Lynch ${ }^{1,2 c}$ \\ ${ }^{1}$ Defence Science and Technology Organisation, Melbourne, Australia \\ ${ }^{2}$ Monash University, Melbourne, Australia \\ a mark.knop@dsto.defence.gov.au, ${ }^{b}$ nick.birbilis@monash.edu, ${ }^{c}$ stan.lynch@dsto.defence.gov.au
}

Keywords: Corrosion Fatigue, Steels, Waveform, Cycle Period, Striations, Nanovoids, Mechanisms, Hydrogen Embrittlement, Adsorption-Induced Dislocation Emission (AIDE)

\begin{abstract}
The processes involved in corrosion fatigue in general are briefly outlined, followed by a brief review of recent studies on the effects of cycle frequency (rise times) and electrode potential on crack-growth rates at 'intermediate' $\Delta \mathrm{K}$ levels for cathodically protected high-strength steels. New studies concerning the effects of fall times and hold times at maximum and minimum loads on crack-growth rates (for $\mathrm{K}_{\max }$ values below the sustained-load SCC threshold) are presented and discussed. Fractographic observations and the data indicate that corrosion-fatigue crack-growth rates in aqueous environments depend on the concentration of hydrogen adsorbed at crack tips and at tips of nanovoids ahead of cracks. Potential-dependent electrochemical reaction rates, crack-tip strain rates, and hydrogen transport to nanovoids are therefore critical parameters. The observations are best explained by an adsorption-induced dislocation-emission (AIDE) mechanism of hydrogen embrittlement.
\end{abstract}

\section{Review of Mechanisms and Some General Aspects of Corrosion Fatigue}

For many materials, embrittling environments (e.g. aqueous, gaseous hydrogen, liquid metals) can increase rates of fatigue crack growth by up to several orders of magnitude compared with the rate in inert environments, with the degree of embrittlement depending on variables such as $\Delta \mathrm{K}$, cycle frequency, and the 'potency' of the environment. In addition, the fracture path and fracturesurface appearance can be markedly different in inert and embrittling environments $[1,2]$. Fatigue in inert environments generally produces transgranular fractures exhibiting 'ductile' striations whereas intergranular or cleavage-like fractures with 'brittle' striations are observed after fatigue in embrittling environments (although brittle striations are sometimes difficult to resolve owing to their shallow profile and post-fracture corrosion/film formation (Fig. 1).

Fatigue crack growth at intermediate $\Delta \mathrm{K}$, for both inert and embrittling environments, generally involves plastic blunting and crack growth during the rising load part of each stress cycle, with resharpening of the crack tip occurring by deformation just behind crack tips during unloading. The difference between crack growth in inert and embrittling environments is that slip is more localised for the latter, resulting in less blunting and greater crack-growth increments for a given crack-tipopening displacement (Fig. 2). This figure, and others like it in the literature [e.g. 3,4], omits an important feature, namely, the formation of nanovoids ahead of cracks. Nanovoid formation at intermediate $\Delta \mathrm{K}$ levels is often overlooked because the presence of nano-scale dimples on fracture surfaces is clearly resolved only when 'state-of-the-art' high-resolution SEM is used [5] or, even better, when TEM is used to examine well-shadowed replicas under ideal conditions [6] (Fig. 3).

The localisation of plasticity and increased crack growth rates in both aqueous and gaseous hydrogen environments for many materials (e.g. Fe, $\mathrm{Mg}, \mathrm{Ni}, \mathrm{Ti}$ ) are generally associated with an effect of atomic hydrogen, chemically or electrochemically generated by dissociation of water, or by dissociation of hydrogen molecules [2]. The precise mechanism of hydrogen embrittlement (for 

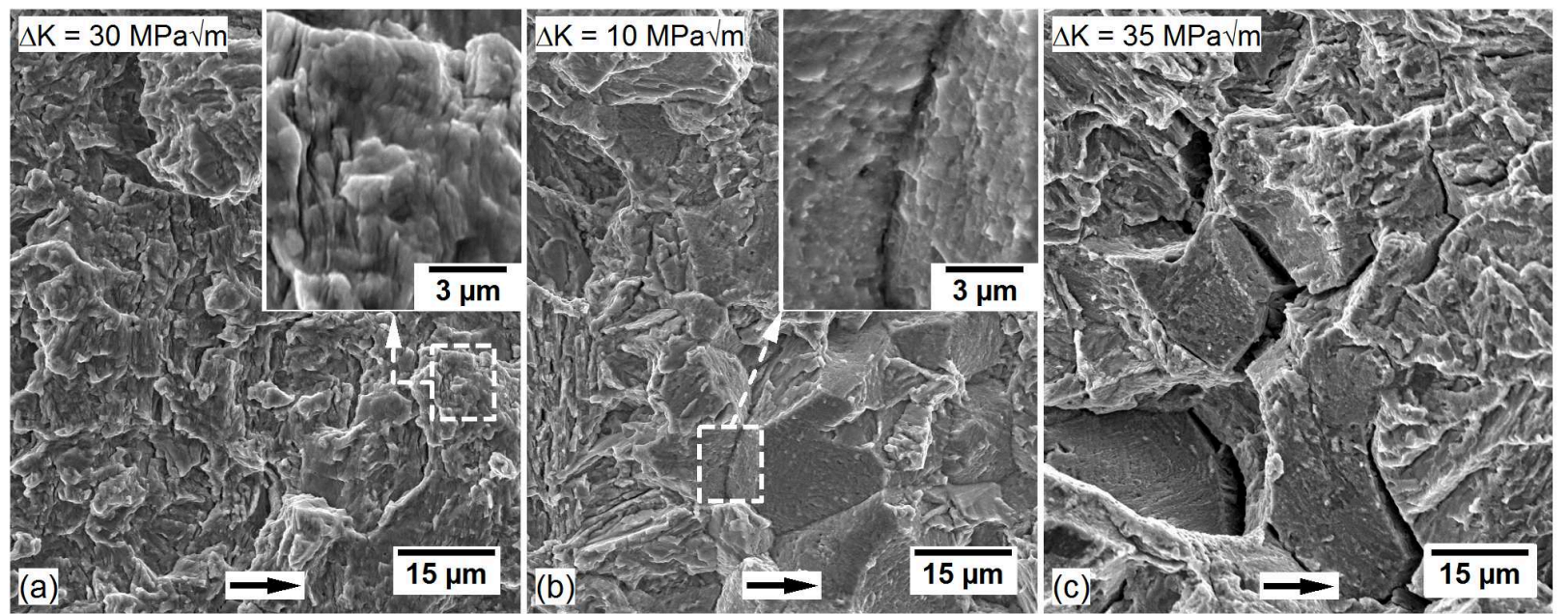

Figure 1: SEMs of fatigue fracture surfaces of a BIS812EMA (750 MPa) steel after crack growth in (a) laboratory air at $20^{\circ} \mathrm{C}$, (b) $\mathrm{NaCl}$ solution with cathodic protection $(-1050 \mathrm{mV}(\mathrm{SCE}))$ at $20^{\circ} \mathrm{C}$, and (c) liquid In-Bi-Sn eutectic (melting point $62^{\circ} \mathrm{C}$ ) at $\sim 70^{\circ} \mathrm{C}$, showing transgranular fracture (exhibiting striations and associated secondary cracks from some of them) for the air environment, and mixed cleavage-like and intergranular facets for the aqueous and liquid-metal environments [7]. Black arrows indicate the direction of crack growth.

(a) Fatigue in 'inert' media
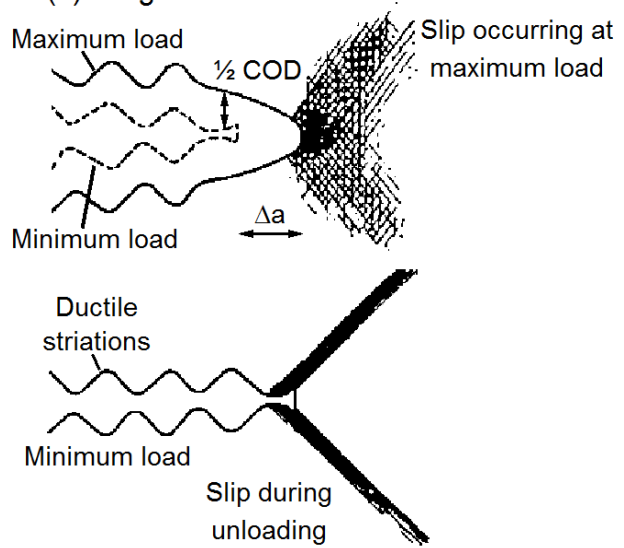

(b) Fatigue in 'embrittling' media
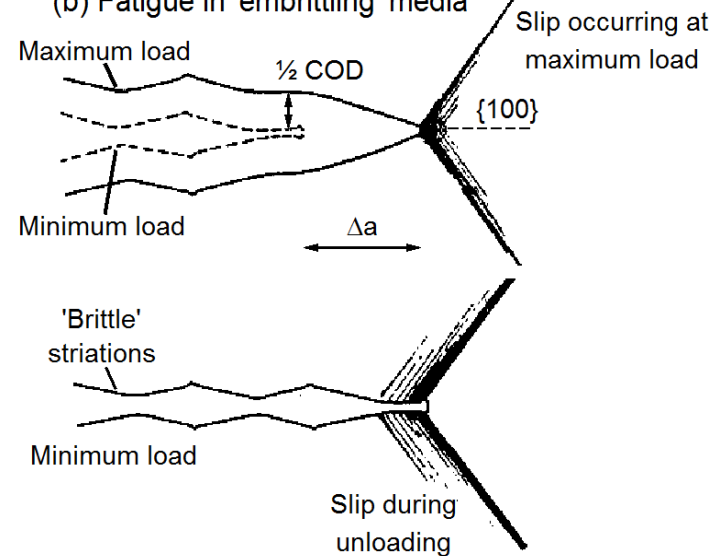

Figure 2: Schematic diagrams illustrating mechanisms of fatigue crack growth and formation of striations at intermediate $\Delta \mathrm{K}$, (a) for inert environments and (b) for embrittling environments [8]. Nucleation and growth of nanovoids (not shown) often occur in highly strained regions just ahead of crack tips in both cases, but slip is more localised for embrittling environments [8].
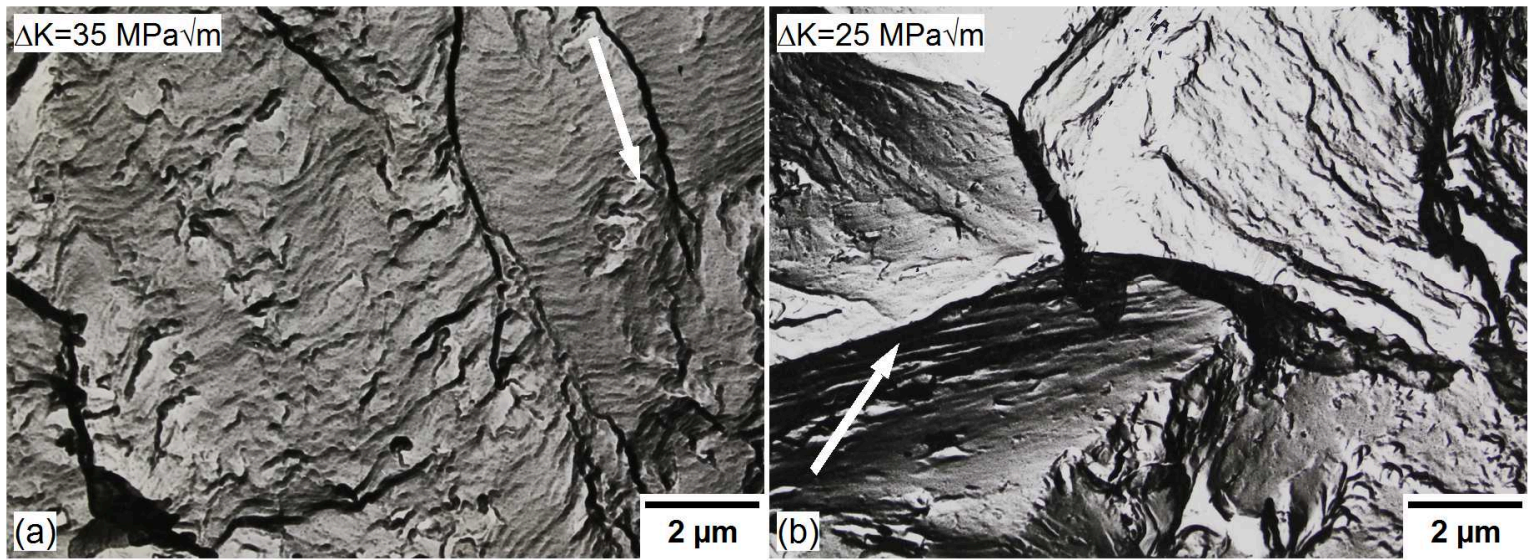

Figure 3: TEM of shadowed carbon replica of a fatigue fracture surface of an ultra-high-strength steel tested in (a) laboratory air, and (b) hydrogen gas, showing ill-defined striations and numerous nano-dimples (50-250 $\mathrm{nm}$ diameter) on (a) transgranular and (b) intergranular facets [9]. White arrows indicate the direction of crack growth. 
materials/conditions where hydrides do not form) is still subject to debate, but it is likely that different mechanisms predominate for different materials and fracture modes [10]. For cleavage-like fractures and intergranular fractures associated with high localised strains, such as occur for corrosion fatigue of cathodically protected steels, an adsorption-induced dislocation-emission (AIDE)/nanovoid-coalescence mechanism probably predominates, with possible minor contributions from a (solute) hydrogen-enhanced localised plasticity (HELP) mechanism and a hydrogen-enhanced decohesion (HEDE) mechanism (Fig. 4) [11]. The similarity in the fracturesurface appearance for fatigue in hydrogen-bearing and in liquid-metal environments (Fig. 1(b), (c)) is one of numerous pieces of evidence that an adsorption mechanism predominates for steels (and some other materials) [10]. Detailed discussion of AIDE, HELP, HEDE, and other mechanisms, and evidence supporting them, can be found in recent reviews $[2,6,10]$.

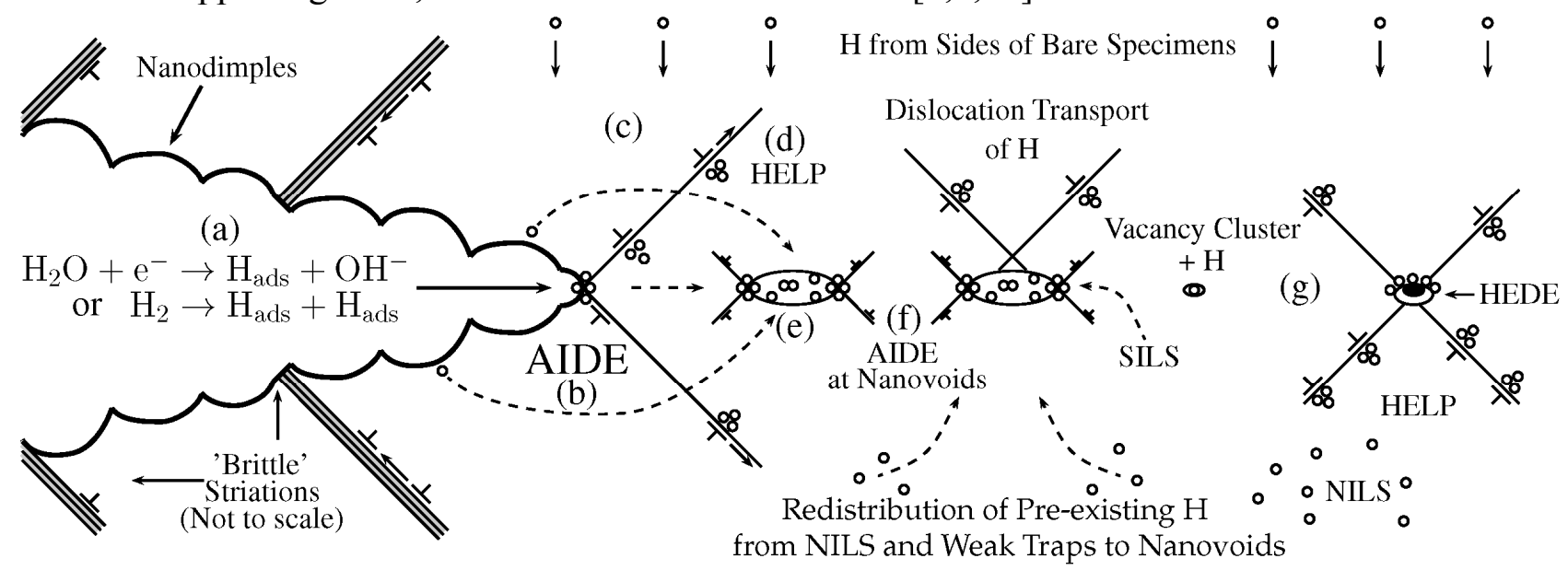

Figure 4: Schematic diagram illustrating processes that could be involved in hydrogen-assisted fatigue-crack growth: (a) Electrochemical or chemical reactions producing hydrogen; (b) Adsorption-induced dislocation-emission (AIDE) from crack tips due to hydrogen on the surface and at surface interstitial lattice sites (SILS) between the first and second atomic layers; (c) Hydrogen diffusion from crack tips and side surfaces to region ahead of cracks; (d) Hydrogenenhanced localised plasticity (HELP) facilitating movement of emitted dislocations away from cracks; (e) Hydrogen diffusion/dislocation transport to nanovoids, and condensation of $\mathrm{H}_{2}$ in nanovoids; (f) AIDE facilitating nanovoid growth; and (g) Nucleation of nanovoids by hydrogeninduced decohesion at particle/matrix interfaces, at intersections of planar slip bands, or at hydrogen-stabilized vacancy clusters [11]. Molecular hydrogen $\left(\mathrm{H}_{2}\right)$ in nanovoids is deeply trapped, will not diffuse away during unloading and, for materials such as steels, will be available for redissociation/adsorption/AIDE during subsequent loading. Preferential adsorption at intergranular crack tips or preferential nanovoid formation along grain boundaries - depending on grain-boundary microstructure, misorientation, and metalloid segregation - could explain why intergranular crack paths are sometimes preferred over transgranular paths.

\section{Corrosion-Fatigue Crack-Growth in Steels: Effects of Rise Time and Other Variables}

Before presenting and discussing new results concerning the effects of hold times at maximum/minimum loads and effects of fall time (for given rise times), it is worth briefly reviewing previous work $[11,12]$ on the effects of rise time, $\Delta \mathrm{K}$, electrode potential, and coatings. Plots of da/dN versus rise time for a given $\Delta \mathrm{K}$ (for positive sawtooth waveforms) show a sigmoidal relationship, with sigmoids displaced upwards and to the right for higher $\Delta \mathrm{K}$ values such that the inflection points of the sigmoids fall on a common transition line (Fig. 5(a)). This effect can account for the shape of plots of $(\log ) \mathrm{da} / \mathrm{dN}$ versus $(\log ) \Delta \mathrm{K}$, which exhibit an effective $\mathrm{da} / \mathrm{dN}$ plateau over an intermediate range of $\Delta \mathrm{K}$, with plateau da/dN values increasing with increasing rise time (Fig. 5(b)). The sigmoidal da/dN versus rise-time plots were explained on the basis that (i) for short 
rise times, surface-reaction kinetics are not fast enough to produce significant concentrations of adsorbed hydrogen at crack tips, and there is insufficient time for re-distribution of pre-existing hydrogen ahead of cracks to the tips of newly generated voids during the cycle or subsequent cycle, so that there is a lower plateau, (ii) for longer rise times, hydrogen concentrations at crack and void tips increase, so that AIDE at both locations is promoted and crack-growth rates increase, and (iii) adsorbed hydrogen concentrations at crack/void tips become 'saturated' after even longer rise-times so that an upper da/dN plateau is observed. For increasing levels of cathodic protection at constant $\Delta \mathrm{K}, \mathrm{da} / \mathrm{dN}$ increased in the transition and upper plateau regimes as more hydrogen became available for adsorption at crack/void tips [11]. For coated specimens, longer rise-times in the transition regime (at $-1050 \mathrm{mV}(\mathrm{SCE})$ ) were necessary to achieve the same crack growth rate as for bare specimens, showing that hydrogen diffusion from the sides (as well as crack tips) played a role [11].

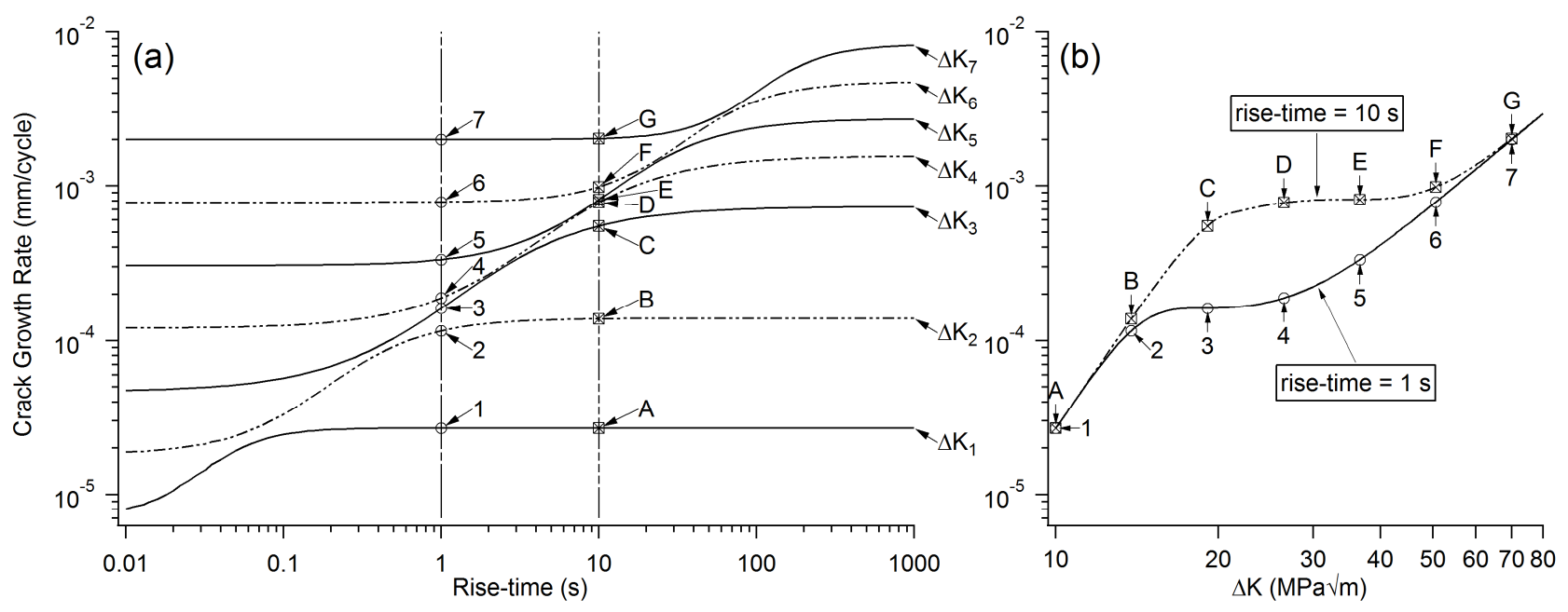

Figure 5: (a) da/dN versus rise-time plots for a positive sawtooth waveform for seven $\Delta \mathrm{K}$ values, with $\mathrm{da} / \mathrm{dN}$ values for each $\Delta \mathrm{K}$ numbered/lettered for two rise times, and (b) construction of da/dN versus $\Delta \mathrm{K}$ plot based on the rise-time data, showing the shape observed experimentally for cathodically protected steel, including the 'plateau' $\mathrm{da} / \mathrm{dN}$ at intermediate $\Delta \mathrm{K}$ [12]. (The plots are based on experimental data, which are similar for a number of steels with strengths in the range of 350-900 MPa $[1,12,13]$.)

\section{Corrosion-Fatigue Crack-Growth Rates in Steels: Effects of Hold and Fall Times}

Experimental Procedure. Tests were carried out using the same experimental procedure as in previous work $[11,12]$. They were carried out under cathodic protection $(-1050 \mathrm{mV}(\mathrm{SCE}))$ in aqueous $3.5 \% \mathrm{NaCl}$ on bare $\mathrm{HY} 80$ steel ( $\sim 600 \mathrm{MPa}$ strength) at a $\Delta \mathrm{K}$ of $30 \mathrm{MPa} \sqrt{\mathrm{m}}$ and an $\mathrm{R}$ ratio of 0.5 for different waveforms, but with the same rise-times of $0.5 \mathrm{~s}$ and $2.5 \mathrm{~s}$ - corresponding to the lower plateau region and the transition region, respectively, of the $\mathrm{da} / \mathrm{dN}$ versus rise-time plot for triangular waveforms with no hold times. For trapezoidal waveforms, the fall time was the same as the rise time. The da/dN values for varying hold-time at (i) maximum load, and (ii) minimum load were compared with those with no hold times, i.e. sawtooth waveforms with varying fall times, for the same cycle period.

Results. Plots of da/dN versus cycle period for the three different waveforms show that there are significant differences, viz., (i) da/dN values for the different waveforms diverge with increasing cycle period, with the $\mathrm{K}_{\max }$-hold waveform having higher $\mathrm{da} / \mathrm{dN}$ than the negative-sawtooth waveform, which had a higher da/dN than the $\mathrm{K}_{\min }$-hold waveform in the intermediate cycle period domain (Fig. 6). Upper plateau da/dN values were observed for all waveforms, but were significantly higher for the $\mathrm{K}_{\max }$-hold waveforms than the negative-sawtooth and $\mathrm{K}_{\min }$-hold waveforms, with this difference decreasing when rise times were longer (Fig. 6). Other tests showed that there were no effects of fall times or hold times at minimum load if the preceding rise time was longer than $\sim 80 \mathrm{~s}$ or preceding hold time at maximum load was longer than $\sim 80 \mathrm{~s}$. 

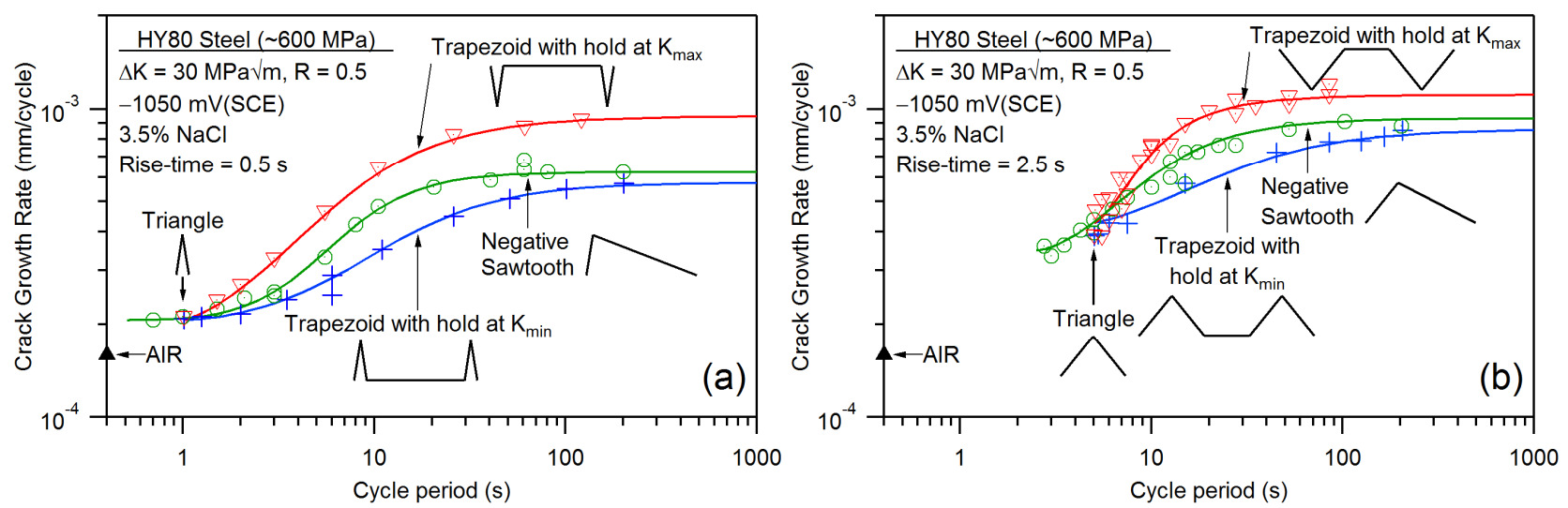

Figure 6: Plots of $\mathrm{da} / \mathrm{dN}$ versus cycle period for the waveforms illustrated: For the same cycle period, hold times at maximum loads promote crack growth more effectively than hold times at minimum load, with negative-sawtooth waveform data lying between the hold-time plots. Comparisons of (a) and (b) show that hold and fall times become less effective at promoting crack growth as the rise time is increased.

Discussion. In discussing the differences in crack growth rates for the different waveforms, one needs to bear in mind that (i) the maximum $\mathrm{K}$ value is well below the sustained-load SCC threshold $\mathrm{K}$ value [14], (ii) there was no effect when rise times were long, and (iii) the comparisons have been made for the same rise time and cycle period such that crack growth promoted by electrochemical reactions/adsorbed hydrogen at the external crack tip during the rise time will be fairly similar, and the time available for hydrogen accumulation in nanovoids ahead of cracks is the same. Thus, some of the differences in the $\mathrm{da} / \mathrm{dN}$ versus cycle-period plots for the different waveforms probably lie in differences in rates of hydrogen accumulation in nanovoids owing to different stress fields, but another difference between the $\mathrm{K}_{\max }$-hold waveform and the others is that it is likely that some crack growth occurs during the $\mathrm{K}_{\max }$ hold time.

Crack-tip strain rates at the start of the $\mathrm{K}_{\max }$ hold time will be high, such that fresh surfaces are produced and, hence, hydrogen generation/AIDE continues to occur at crack tips. However, strain rates will decay with increasing hold time so that crack growth slows down and then stops when the strain rate decreases below a critical value - which (partially) explains why da/dN reaches a plateau value after a certain hold time, but one that is higher than the other two waveforms. An upper plateau $\mathrm{da} / \mathrm{dN}$ (for all three waveforms) also arises because hydrogen concentrations in nanovoids ahead of cracks reach a 'saturation' level, as discussed for the rise-time data.

Saturation of hydrogen in voids (and reaching the upper plateau da/dN) takes longer to achieve at $\mathrm{K}_{\mathrm{min}}$-hold waveforms than negative-sawtooth waveforms because stress fields around crack tips are compressive for longer for $\mathrm{K}_{\min }$-hold waveforms. The $\mathrm{K}_{\max }$-hold waveform should result in the fastest rate of hydrogen accumulation in nanovoids, owing to hydrostatic tensile stresses around cracks and, hence, the shortest time (or cycle period) to reach saturated hydrogen levels/upper plateau da/dN. However, this is not apparent from the plots in figure 6 because transient crack growth also occurs during the hold time.

Hold and fall times become less effective at promoting crack growth as the rise time increases, probably because (i) crack-tip strain rates at the start of the $\mathrm{K}_{\max }$ hold time should be lower since crack-tip-opening strain will have been accommodated more by larger crack-growth increments during the rise time, and (ii) hydrogen concentrations at nanovoids will be higher at the end of longer rise times (and will already be saturated after very long rise times). Similarly, fall times and hold times at minimum load will have no effect on crack growth if nanovoids have already become saturated during preceding rise times or hold times at maximum loads. 


\section{Conclusions}

Increasing rise time is most effective in promoting crack growth, but longer hold times at maximum and minimum loads and longer fall times can also promote crack growth if rise times are short. The effects of these variables, as well as electrode potential and coatings on side surfaces, can best be explained in terms of an adsorption-induced dislocation emission (AIDE)/nanovoid-growth mechanism of hydrogen-assisted cracking, with kinetics largely controlled by surface-reaction rates at external crack tips and rates of hydrogen accumulation at nanovoids ahead of cracks, as illustrated schematically in figure 4 of the present paper, and discussed in detail elsewhere [11].

\section{References}

[1] R.P. Gangloff, Corrosion fatigue crack propagation in metals, in: R.P. Gangloff, M.B. Ives (Eds.), Environment-Induced Cracking of Metals, NACE, Houston, 1988, pp. 55-109, and references therein.

[2] S.P. Lynch, Mechanistic and fractographic aspects of stress-corrosion cracking (SCC), pp. 3-89, and Hydrogen embrittlement phenomenon and mechanisms, pp.90-130, in: V.S. Raja, T. Shoji (Eds.), Stress Corrosion Cracking, Woodhead Publishing Limited, Cambridge, 2011. Republished in Corrosion Reviews 30 (2012) 63-104 and 105-123, and see references therein.

[3] S. Suresh, Fatigue of Materials, second ed., Cambridge University Press, Cambridge, 1988.

[4] R. Pippan, C. Zelger, E. Gach, C. Bichler, H. Weinhandl, On the mechanism of fatigue crack propagation in ductile metallic materials, Fatigue Fract. Engng Mater. Struct. 34 (2010) 1-16.

[5] T. Neeraj, R. Srinivasan, Ju Li, Hydrogen embrittlement of ferritic steels: observations on deformation microstructure, nanoscale dimples and failure by nanovoiding, Acta Mater. 60 (2012) $5160-5171$.

[6] S.P. Lynch, Environmentally assisted cracking: overview of evidence for an adsorption-induced localised-slip process, Acta Metall. 36 (1988) 2639-2661.

[7] M. Knop, S.P. Lynch, Unpublished research.

[8] S.P. Lynch, Mechanisms of fatigue and environmentally assisted fatigue, in: J.T. Fong (Ed.), Fatigue Mechanisms, ASTM STP 675, 1979, pp. 174-213.

[9] S.P. Lynch, N.E. Ryan, Mechanisms of hydrogen embrittlement - crack growth in a low alloy ultra-high-strength steel under cyclic and sustained stresses in gaseous hydrogen, ARL-MatReport-103, Defence Science and Technology Organisation, 1978.

[10] S.P. Lynch, Mechanisms and kinetics of environmentally assisted cracking: current status, issues, and suggestions for future work, Metall. and Mater. Trans. A 44A (2012) 1209-1229.

[11] M. Knop, N. Birbilis, S.P. Lynch, Corrosion fatigue cracking in high-strength steels: effects of cycle frequency, waveform, and potential, in: B.P. Somerday, P. Sofronis (Eds.), Hydrogen Materials Interactions, ASME, New York, 2013, to be published

[12] M. Knop, J. Heath, Z. Sterjovski, S.P. Lynch, Effects of cycle frequency on corrosion-fatigue crack growth in cathodically protected high-strength steels, Procedia Engng 2 (2010) 1243-1252.

[13] R. Krishnamurthy, C.N. Marzinsky, R.P. Gangloff, Microstructure and yield strength effects on hydrogen environment fatigue of steels, in: N.R. Moody, A.W. Thompson (Eds.), Hydrogen Effects on Material Behavior, The Minerals, Metals \& Materials Society, 1990, pp. 891-908.

[14] R.A. Hays, D.A. Davis, D.M. Aylor, Stress corrosion cracking of naval structural steels, in: Tri-Service Corrosion Conference, Office of Naval Research, Wrightsville Beach, NC, 1997. 